\title{
Metabolic and bariatric surgery
}

\author{
Daniel B. Jones
}

Beth Israel Deaconess Medical Center, Harvard Medical School, Boston, MA 02215, USA.

Correspondence to: Prof. Daniel B. Jones, Beth Israel Deaconess Medical Center, Harvard Medical School, Boston, MA 02215, USA.E-mail: djones1@bidmc.harvard.edu

How to cite this article: Jones DB. Metabolic and bariatric surgery. Mini-invasive Surg 2021;5:4 http://dx.doi.org/10.20517/2574-1225.2020.116

Received: 15 Dec 2020 Accepted: 16 Dec 2020 Published: 7 Jan 2021

Academic Editor: Giulio Belli Copy Editor: Miao Zhang Production Editor: Jing Yu

Obesity is a disease causing multiple comorbid health conditions such as type 2 diabetes, hypertension, obstructive sleep apnea, back pain, and cancers. Weight loss improves overall health and quality of life. When diets, exercise, and behavioral changes are not enough, weight loss operations can help patients lose 100 pounds or more, reverse associated health problems, and increase longevity.

In the past year, COVID-19 has affected over 1.6 million people worldwide, with over 300,000 deaths in the Unites States alone. During this time, we have learned a lot about inflammation and response to COVID-19. We have known that obesity creates a chronic inflammatory state and that it contributes to many other diseases such as diabetes. However, during the COVID-19 pandemic, we have witnessed that patients who are overweight and with weight-related illness are at higher risk of death after exposure to the coronavirus. With a vaccine released this week, we are all hopeful for immunity. We are also more mindful than before about the effects of obesity on health and wellbeing.

The Mini-invasive Surgery Journal sought to have a Special Issue for "Metabolic and Bariatric Surgery." This is a bold initiative with so many other outlets to publish manuscripts on the topic. When the Journal asked me to co-edit the volume, I was intrigued. We recruited authors from around the world to share their observations, science, and reviews of the literature. I am very grateful to those authors who managed to contribute despite the pandemic considering the added demands on providers.

In this volume, Dr. Rami Lutfi ${ }^{[1]}$ summarized the current status of metabolic surgery. He emphasized that bariatric operations are treating diabetes. He reviewed the STAMPEDE trial, 2nd Diabetes Surgery Summit, and guidelines from the American Diabetes Association. He also reviewed the choice of different operations.

cc) (i) The Author(s) 2021. Open Access This article is licensed under a Creative Commons Attribution 4.0 International License (https://creativecommons.org/licenses/by/4.0/), which permits unrestricted use, sharing, adaptation, distribution and reproduction in any medium or format, for any purpose, even commercially, as long as you give appropriate credit to the original author(s) and the source, provide a link to the Creative Commons license, and indicate if changes were made. 
For lower weights, endoscopic therapy is evolving. Mlabasati et al. ${ }^{[2]}$ at BIDMC shared lessons learned setting up an Endoscopic Bariatric multidisciplinary program to provide intragastric balloon and endo plication for obesity. Dr. Aurora Pryor ${ }^{[3]}$ reviewed the endoscopic approaches to treating complications of bariatric surgery. She noted a 4\%-10\% complication rate in the first month after bariatric surgery. She went on to describe endoscopic treatments for complications of bleeding, strictures, ulcer, reflux, and weight regain. With endoscopic techniques of injection, clipping, stents, balloons, and Stretta, the endoscope is a valuable adjunct to providing comprehensive care.

Long-term complications of malabsorption may include vitamin deficiency such as vitamin B12, iron, vitamin D, calcium, and folate. Çalapkorur and Küçükkatirci ${ }^{[4]}$ from Nevsehir, Turkey described each deficiency in detail. This review is a must read for all providers caring for postoperative bariatric surgery patients. Vitamin deficiencies, such as in thiamine, if go unrecognized, can lead to serious and irreversible neurological problems. Early identification and early treatment are crucial.

The sleeve gastrectomy and Roux-en-Y gastric bypass (RYGB) are the most commonly performed weight loss operations in the world. Fontan et al. ${ }^{[5]}$ stated they prefer RYGB for patients with gastro-esophageal reflux disease (GERD). However, other procedures such as the one anastomosis gastric bypass (OAGB) have been growing in popularity despite the concern for bile reflux. The Paris Descartes Faculty of Medicine studied reflux, Barrett's esophagus, and esophageal cancer ${ }^{[6]}$. They shared their findings and concluded that the OAGB operation appears to be safe in an animal model.

The devil is in the details with any operation. Aktokmakyan et al. ${ }^{[7]}$ from Istanbul, Turkey reviewed the technical steps to a perfect sleeve gastrectomy. They cited the literature including the 5th International Consensus Conference. Their paper includes high resolution intraoperative photographs. While I also use a $36 \mathrm{fr}$ Bougie to size my sleeve, I disagree that the anastomosis must be checked by methylene blue or endoscopy. I also no longer place a drain. Today, many providers utilize an ERAS protocol that limits narcotics and shortens hospital stay.

Fewer than $1 \%$ of the patients who meet criteria for weight loss surgery actually have an operation. Aly et al. ${ }^{[8]}$ from Boston Medical Center reviewed psychological, social, and cultural barriers to seeking treatment and getting care.

The Special Issue "Metabolic and Bariatric Surgery" covers the essentials of technique and perioperative care. The Special Issue is also the first to report the use of intra-aortic balloon pump (IABP) ${ }^{[9]}$ during sleeve gastrectomy and complication of patulous eustachian tube $(\mathrm{PET})^{[10]}$ after sleeve gastrectomy.

Bariatric surgeons have known for a long time that metabolic operations reverse many comorbid conditions. Society is learning that obesity and related conditions may be life-threatening and metabolic operations lifesaving in the era of COVID-19.

\section{DECLARATIONS}

\section{Authors' contributions}

The author contributed solely to the article.

\section{Availability of data and materials}

Not applicable.

\section{Financial support and sponsorship}

None. 


\section{Conflicts of interest}

The author declared that there are no conflicts of interest.

\section{Ethical approval and consent to participate}

Not applicable.

\section{Consent for publication}

Not applicable.

\section{Copyright}

(c) The Author(s) 2021.

\section{REFERENCES}

1. Veilleux E, Lutfi R. Metabolic and bariatric surgery: diabetes - a decade of discovery. Mini-invasive Surg 2020;4:4.

2. Mlabasati J, Bilal M, Cohen J. Early lessons on assembling a center for bariatric endoscopy. Mini-invasive Surg 2020;4:42.

3. Ardila-Gatas J, Pryor A. Endoscopic approach for the treatment of bariatric surgery complications. Mini-invasive Surg 2020;4:16.

4. Çalapkorur S, Küçükkatirci H. Vitamin deficiencies and prevention methods after bariatric surgery. Mini-invasive Surg 2020;4:15.

5. Fontan FM, Carroll RS, Thompson D, Lehmann RK, Smith JK, Nau PN. Current management of gastroesophageal reflux disease in the obese population - a review of the literature. Mini-invasive Surg 2020;4:29.

6. M'Harzi L, Bruzzi M, Chevallier JM, Douard R. One anastomosis gastric bypass and esojejunostomy in rats: surgical techniques. Miniinvasive Surg 2019;3:27.

7. Aktokmakyan TV, Gungor O, Sumer A. Technical details of laparoscopic sleeve gastrectomy. Mini-invasive Surg 2020;4:23.

8. Aly S, Hachey K, Pernar LIM. Gender disparities in weight loss surgery. Mini-invasive Surg 2020;4:21.

9. Narvaez A, Perez JE, Castro M, Seymour KA. Use of an intra-aortic balloon pump during laparoscopic sleeve gastrectomy. Mini-invasive Surg 2020;4:31.

10. Larionova E, Jalisi SM, Jones DB. Hearing voices and strange noises after sleeve gastrectomy. Mini-invasive Surg 2020;4:59. 